Proceedings of the XXIII Conference on Applied Crystallography, Krynica Zdrój, Poland, September 20-24, 2015

\title{
The Moment Series Expansion for Quasicrystal with Phason Disorder
}

\author{
I. Buganski, R. Strzalka And J. Wolny \\ AGH University of Science and Technology, Faculty of Physics and Applied Computer Science, \\ al. A. Mickiewicza 30, 30-059 Krakow, Poland
}

\begin{abstract}
The novel method for structural analysis of quasicrystals with phason flips is presented. The correction for diffraction peaks' intensities can be made within average unit cell approach by modification of the statistical distribution of atomic positions. Characteristic function of the distribution expanded into moment series, involving only even moments, estimates the envelope function and therefore the flip ratio can be evaluated.
\end{abstract}

DOI: 10.12693/APhysPolA.130.833

PACS/topics: 61.44.Br, 61.05.cp, 61.43.-j

\section{Introduction: the statistical approach}

The statistical approach known as the average unit cell (AUC) method [1] is the alternative to the higher dimensional description of quasicrystalline structures. The basic concept involves calculation of the statistical distribution of atomic positions within periodic, reference lattice framework. Every atomic position $x$ is projected onto two periodic lattices with lattice constants $\lambda_{k}=2 \pi / k_{0}$ and $\lambda_{q}=2 \pi / q_{0}$, where $k_{0}$ - length of the reciprocal space basis vector, $q_{0}$ - length of the modulation vector. Each position $x$ in the periodic lattice has coordinates $u, v$ as follows:

$$
\begin{aligned}
& u=x \bmod \left(\lambda_{k}\right), \quad u \in\left[0, \lambda_{k}\right), \\
& v=x \bmod \left(\lambda_{q}\right), \quad v \in\left[0, \lambda_{q}\right) .
\end{aligned}
$$

The distribution of coordinates $P(u, v)$ is obtained. It can be shown that structure factor $F(k)$ for the scattering vector $k=n k_{0}+m q_{0}, n, m \in Z$ is dependent on the distribution $P(u, v)[1]$ :

$$
F(k)=\int_{0}^{\lambda_{k}} \int_{0}^{\lambda_{q}} P(u, v) \exp \left(\mathrm{i} n k_{0} u+\mathrm{i} m q_{0} v\right) \mathrm{d} u \mathrm{~d} v \text {. }
$$

For quasicrystals with scaling $\tau=(1+\sqrt{5}) / 2$, reciprocal space vectors ratio reads $k_{0} / q_{0}=\tau$. The $u$ and $v$ coordinates are linearly dependent

$$
v=-\tau^{2} u \text {. }
$$

Applying of the formula (3) in (2) simplifies the structure factor definition reading

$$
F(k)=\int_{0}^{\lambda_{k}} P(u) \exp (\mathrm{i} u w) \mathrm{d} u=F(w),
$$

where $P(u)$ is the marginal distribution of the $P(u, v)$ and $w=k-k_{0} m \sqrt{5}$ is the reduced scattering vector. Equation (4) states that structure factor is the Fourier transform of the distribution $P(u)$. Function $F(w)$ is called the envelope function [2]. The diffraction pattern consists of peaks indexed by two integers $n, m \in Z$. According to (4) peaks with the same value of index $m$ belong to the common envelope in $k$ space. This envelope must be translated by vector $k_{1}=k_{0} m \sqrt{5}$ in order to embrace all peaks (Fig. 1, dashed line). In the space

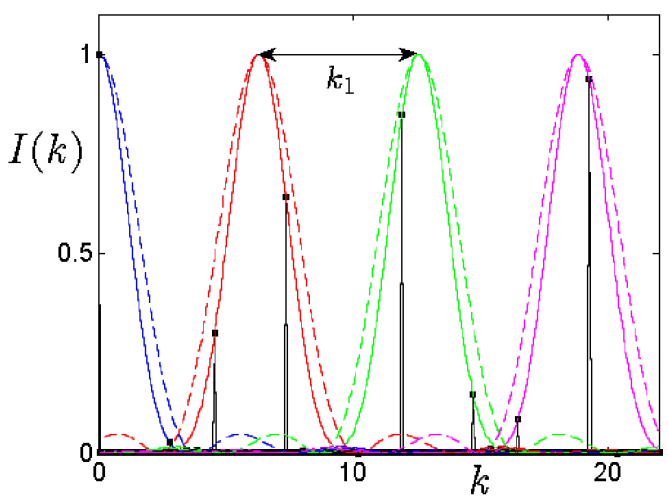

Fig. 1. The diffraction pattern of Fibonacci chain with $\alpha=0.4$ of phason flips in the structure. Four envelopes are shown (solid line). Dashed line represent envelope for Fibonacci chain without flips.

of the so-called reduced vector $w$ all peaks are grouped into one curve [3].

\section{Phason disorder}

The Fibonacci chain (FC) is a well-known example of quasicrystalline structure with $\tau$-scaling [4]. The structure is composed of two bonds: long $L$ and short $S$. The ratio of lengths $L / S=\tau$. The phason disorder $[5,6]$ can be simply introduced to the structure of the Fibonacci chain by rearrangement of two subsequent tiles $L$ and $S[7,8]$. Probability of single flip will be denoted as $\alpha$. Reshuffle of tiles is done by shifting the position of middle node lying between outer nodes.

The statistical distribution $P(u)$ for the FC can be constructed. The distribution is uniform [9] but it is nonzero only in the part of the domain [10] (Fig. 2, dashed line). The statistical distribution is reshaped after application of phason flips (Fig. 2) differently for $L S \rightarrow S L$ and $S L \rightarrow L S$ flips. Relation (3) is preserved. The distribution for two types of flips differs but it can be shown, that in the space of the reduced wave vector $w$ the difference influences only the global phase, which is irrelevant when calculating intensities. In further analysis we focus on flips $L S \rightarrow S L$ only. 
TABLE I
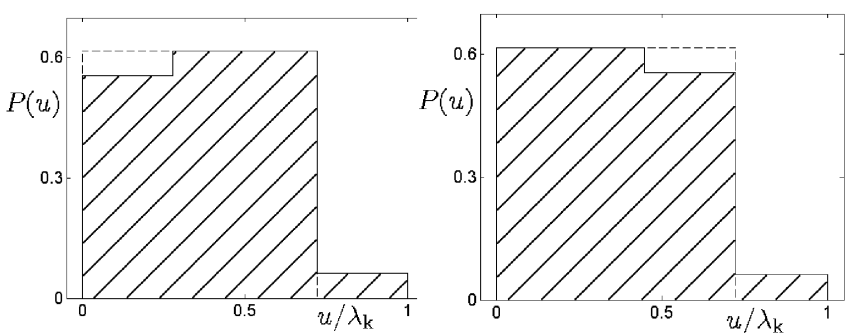

Fig. 2. The marginal distribution $P(u)$ for the FC with flip ratio $\alpha=0.1$ for flips $L S \rightarrow S L$ (left) and $S L \rightarrow L S$ (right). In both cases dashed line shows the boundary of ideal statistical distribution without flips.

According to results presented in [11] the commonly used correction for phason disorder [12] (perpendicularspace Debye-Waller factor) does not properly handle changes of peaks' intensities in small peaks regime. A novel concept was proposed based on the estimation of the envelope function by expanding it to moment series. It can be seen (Fig. 1) that flips change the shape of the envelope function. Having the analytic formula for the envelope function it would be possible to calculate the structure factor and by this retrieve the atomic positions. Equation (4) is by definition the characteristic function of the marginal distribution $P(u)$ and always can be approximated by its moment expansion.

\section{Model analysis}

Due to the Friedel theorem the observable diffraction pattern is always centrosymmetric. According to this the methodology presented in [11] can be simplified and can limit calculation to only parameters for even powers. The contribution from imaginary part can be neglected. The intensity of the peak can be expressed as

$$
I(w)=\sum_{j=0}^{N} a_{2 j} w^{2 j}+O\left(w^{2 N}\right),
$$

where $a_{2 j}$ are parameters to be fitted. Coefficients $a_{2 j}$ depend on the even moments only. Equation (5) is fitted against the diffraction pattern calculated upon cluster of 30000 positions of $\mathrm{FC}$ with three different flip ratio: $\alpha=0.1, \alpha=0.2, \alpha=0.4$. Maximal number of fitted parameters in each case is equal to nine. The region of fitting was chosen to contain values of reduced vector $w$ in domain [-10 10]. The value of $R$-factor was calculated after every fitting procedure. Collected values of $R$-factor presented in Table I shows perfect convergence starting from six fitted parameters. Additional parameters of fit do not significantly improve value of $R$-factor.

The flip ratio can be estimated with the value of $a_{2}=$ $\left\langle u^{2}\right\rangle$ - the second moment of the distribution $P(u)$. The value of the second moment depend on the flip ratio (Fig. 3). The theoretical dependence is as follows [11]:

$$
\left\langle u^{2}\right\rangle=\frac{-12 \alpha^{2}+12 \alpha+\tau^{4}}{12 \tau^{2}}
$$

Equation (6) assumes that $P(u)$ is centrosymmetric, which means whole distribution (Fig. 2, top) is shifted
The value of $R$-factor for three different phason flips ratio as a function of number of fitted parameters $(N)$.

\begin{tabular}{c|c|c|c}
\hline \hline \multirow{2}{*}{$N$} & \multicolumn{3}{|c}{$R$-factor [\%] } \\
\cline { 2 - 4 } & $\alpha=0.1$ & $\alpha=0.2$ & $\alpha=0.4$ \\
\hline 2 & 93.2 & 86.7 & 110 \\
3 & 41.3 & 34.9 & 53.5 \\
4 & 8.88 & 4.98 & 18.2 \\
5 & 0.82 & 0.38 & 1.17 \\
6 & 0.11 & 0.23 & 0.22 \\
7 & 0.02 & 0.03 & 0.15 \\
8 & 0.02 & 0.03 & 0.07 \\
9 & 0.00 & 0.03 & 0.05
\end{tabular}

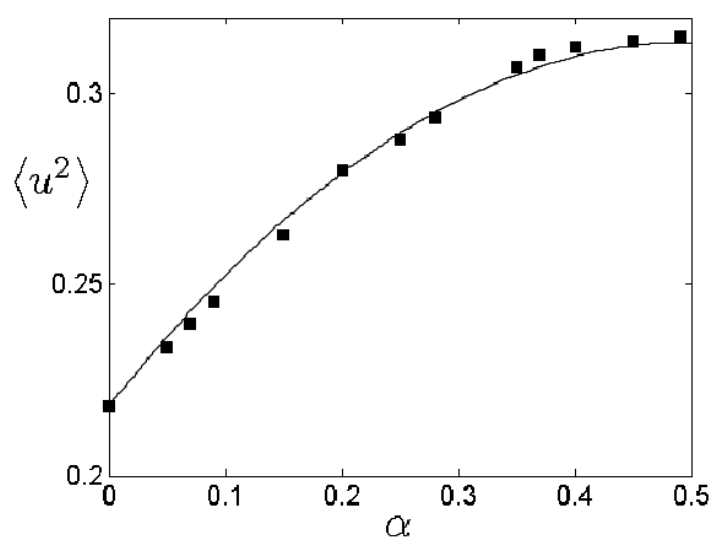

Fig. 3. The value of the second moment of the marginal distribution (black squares) compared with theoretical dependence on flip ratio (solid line).

by vector $[-\langle u\rangle, 0]$. The translation does not influence peaks' intensities. It only affects the value of global phase which is irrelevant. Figure 3 shows values of $\left\langle u^{2}\right\rangle$ obtained from fitting procedure for different ratio of phason flips using nine parameters compared with the theoretical curve. The agreement is satisfying.

\section{Conclusions}

The moment series expansion of the envelope function with only even powers in the series was used to estimate the phason flip ratio in the Fibonacci chain. The latter procedure reduces number of parameters to be fitted in comparison to [11] giving comparable quality of convergence based on $R$-factor values. The value of the second moment of the distribution allows for estimating flip ratio based on the theoretical curve. The flip ratio can be uniquely determined due to injective dependence of the second moment on the flip ratio.

\section{Acknowledgments}

Authors kindly acknowledge financial support from Marian Smoluchowski Krakow Research Consor- 
tium "Matter-Energy-Future" under KNOW dotation (I.B.) and National Science Center, Poland, under grants no. DEC-2013/11/B/ST3/03787 and 2014/13/N/ST3/03776 (R.S.).

\section{References}

[1] J. Wolny, Philos. Mag. A 77, 395 (1998).

[2] J. Wolny, P. Kuczera, R. Strzalka, Appl. Phys. Lett. 106, 131905 (2015).

[3] J. Wolny, B. Kozakowski, P. Kuczera, R. Strzalka, A. Wnek, Israel J. Chem. 51, 1275 (2011).

[4] M. Senechal, Quasicrystals and Geometry, Cambridge University Press, Cambridge 1995.

[5] M. de Boissieu, Philos. Mag. 88, 2295 (2008).
[6] T. Janssen, O. Radulescu, Ferroelectrics 305, 179 (2004).

[7] H. Lipp, M. Engel, S. Sonntag, H.-R. Trebin, Phys. Rev. B 81, 064302 (2010).

[8] Quasicrystal: Structure and Physical Properties, Ed. H.-R. Trebin, Wiley-VCH, Weinheim 2006.

[9] J. Wolny, Acta Crystallogr. A 54, 1014 (1998).

[10] P. Buczek, L. Sadun, J. Wolny, Acta Phys. Pol. B 36, 919 (2005).

[11] I. Buganski, R. Strzalka, J. Wolny, Phys. Status Solidi B 253, 450 (2016).

[12] P.A. Bancel, Phys. Rev. Lett. 63, 2741 (1989). 